\title{
CONOCIMIENTOS Y PRÁCTICAS ASOCIADAS A TRANSMISIÓN DE TRIQUINELOSIS, HANTA Y EQUINOCOCOSIS QUÍSTICA ${ }^{1}$
}

\section{KNOWLEDGE AND PRACTICES ASSOCIATED WITH TRANSMISSION OF TRICHINELLOSIS, HANTA AND CYSTIC EQUINOCOCCOSIS}

\author{
María Alvear Almendras* \\ Claudia Troncoso Muñoz ${ }^{* *}$ \\ Florencia Lastarria CUEVAS ${ }^{* * *}$ \\ Félix Aliaga Russel ${ }^{* * * *}$ \\ Juan Pablo Concha Rosales ${ }^{* * * * *}$ \\ Luis Henríquez Alvear ${ }^{* * * * *}$ \\ Catalina Rivera GutiéRReZ ${ }^{* * * * * *}$
}

\section{RESUMEN}

Objetivo: Asociar el nivel de conocimiento y prácticas (hábitos y costumbres) responsables de la transmisión de Triquinelosis, Síndrome Cardiopulmonar por Hantavirus y Equinococosis quística (Hidatidosis), en habitantes de los sectores rurales. Material y método: Estudio descriptivo correlacional de corte transversal en el que se aplicó una encuesta a 149 habitantes residentes en el área rural de las localidades de Curacautín, Lonquimay y Melipeuco, a través de muestreo no probabilístico por conveniencia, durante los meses de diciembre 2013 a enero 2014. Resultados: La población posee mejores conocimientos de Triquinelosis y Hanta que Hidatidosis, $64,9,72,8$ y 39,3\%, respectivamente; los hábitos y costumbres para Hanta e Hidatidosis alcanzan mejores prácticas ( $84 \%$ cada una) que para Triquinelosis $(69,5 \%)$; la población reconoce correctamente reservorio, fuente de infección y mecanismos de transmisión en Triquinelosis y Hanta, pero en menor grado Hidatidosis; respecto de los hábitos y costumbres, aun desconociendo los fundamentos teóricos, reportan buenas prácticas, aunque mantienen algunas que favorecen la transmisión de infecciones predominantemente en Triquinelosis. Conclusión: El nivel de conocimiento no asegura buenas prácticas, además los conocimientos y prácticas siguen un patrón empírico más que cognitivo, respaldados por aspectos culturales y del entorno. Esto sugiere mayor participación de los equipos interdisciplinarios en comunidades aisladas para fortalecer la promoción, educación y refuerzo de las buenas prácticas de acuerdo con sus condiciones sociales e incentivar la adherencia a conductas que reduzcan el riesgo de la transmisión de zoonosis.

Palabras clave: Zoonosis; Conocimientos, Actitudes y Prácticas en salud; Triquinelosis; Infecciones por Hantavirus; Equinococosis; Enfermería Rural.

\footnotetext{
${ }^{1}$ Financiado por Universidad de La Frontera. Proyecto DIUFRO Nº D12-0020.

*Enfermera, Universidad de La Frontera, Temuco, Chile. Email: maria.alvear@ufrontera.cl. Autor de correspondencia.

** Tecnólogo Médico, Universidad Autónoma de Chile, Temuco, Chile. Email troncosomunozc@gmail.com

${ }^{* * *}$ Enfermera, Universidad de La Frontera, Temuco, Chile. Email: florencia.lastarria@ufrontera.cl

${ }^{* * * *}$ Médico Cirujano, Universidad de La Frontera, Temuco, Chile. Email: felix.aliaga@ufrontera.cl

${ }_{* * * * *}^{*}$ Estadístico, Universidad Austral, Temuco, Chile. Email: juanpabloconcha@gmail.com

${ }^{* * * * * *}$ Profesor de Educación Física, Universidad de La Frontera, Temuco, Chile. Email: luis.henriquez@ufrontera.cl

${ }_{* * * * * *}^{*}$ Profesora de Educación Física, Universidad Católica de Temuco, Temuco, Chile. Email: catar_g@hotmail.com
} 


\section{ABSTRACT}

Objective: To associate the level of knowledge and practices (habits and customs) responsible for the transmission of trichinellosis, Hanta and cystic echinococcosis (hydatidosis), among rural population. Material and method: Cross-sectional correlational descriptive study in which 149 residents of the rural areas of Curacautín, Lonquimay and Melipeuco were surveyed, using non-probability convenience sampling from December 2013 to January 2014. Results: The population has better knowledge of trichinellosis and Hanta than hydatidosis, $64.9 \%, 72.8$ and $39.3 \%$ respectively; they also have better practices regarding Hanta and hydatidosis (84\% each) than regarding trichinellosis $(69.5 \%)$. The population recognizes correctly the reservoir, source of infection and transmission mechanisms of trichinellosis and Hanta, and to a lesser degree hydatidosis; they report good practices regarding habits and customs, even ignoring theoretical foundations, but maintain some practices that favor the transmission of infections related to trichinellosis. Conclusion: The level of knowledge does not ensure good practices. Additionally, knowledge and practices follow an empirical rather than cognitive pattern, rooted on cultural and environmental aspects. This suggests greater participation of interdisciplinary teams in isolated communities to strengthen the promotion, education and reinforcement of good practices in accordance with their social conditions and to encourage adherence to behaviors that reduce the risk of the transmission of zoonoses.

Key words: Zoonoses, Health Knowledge, Attitudes, Practice; Trichinellosis; Hantavirus Infections; Echinococcosis; Rural Nursing.

Fecha recepción: 20/12/2017 Fecha aceptación: 26/10/2018

\section{INTRODUCCIÓN}

Los seres humanos a nivel mundial sufren de variadas enfermedades zoonóticas, algunas de las cuales pueden ser mortales ${ }^{(1)}$. La Triquinelosis, denominada también como triquinosis, presenta una ocurrencia mundial de 10.000 casos anuales $^{(2)}$. En Vietnam, año 2017, se reportaron 114 personas afectadas con 8 muertes $^{(3)}$. Esta infección ha sido reportada en 55 países alrededor del mundo, alcanzando 65.818 casos entre el periodo 1986 a $2009^{(4)}$. En Chile entre 2005 y 2015 se reportaron 258 personas principalmente en la Región Metropolitana entre 30 y 49 años $^{(5)}$.

La Equinococosis quística, que provoca hidatidosis, es considerada una enfermedad emergente a nivel mundial, prevalente en Eurasia, Norte y Este de África, Australia y Sudamérica ${ }^{(6)}$. En Asia Central se le considera una de las zoonosis caninas más prevalentes ${ }^{(7)} \mathrm{y}$ un problema de salud pública grave en China ${ }^{(8)}$. En Rusia se estima una tasa anual de 5 casos/100.000 habitantes, con un $30-50 \%$ de las ovejas infectadas y el $5 \%$ de pe- rros portadores ${ }^{(9)}$. En Chile, en el periodo 19992009 , la incidencia de cirugías por Equinococosis quística humana alcanzó un rango entre 3,6 a 8,4 casos $^{(10)}$ y actualmente se reporta una tasa de incidencia, a nivel nacional, de 1,48 casos por cada 100.000 habitantes, incluso se estima mayor debido a la subnotificación de $\operatorname{casos}^{(11)}$.

Respecto al Hanta virus, especie viral perteneciente a la familia Bunyaviridae, es endémica de Argentina y Chile ${ }^{(12)}$ y se manifiesta con Síndrome Cardiopulmonar por Hantavirus (SCPH), asociado a Virus Andes ${ }^{(13)}$. Específicamente en Chile, ha evolucionado como un problema emergente de salud pública, provocado por factores medioambientales responsables de su evolución epidemiológica ${ }^{(14)}$.

Las causas de transmisión de estas zoonosis son diversas ${ }^{(15)}$. En la Triquinelosis, se trata de una infección parasitaria provocada por diferentes especies pertenecientes al género Trichinella, asociada al consumo de carne mal cocida de porcinos domésticos ${ }^{(16)}$ y silvestres en menor grado. Reportes en Rumania indican que, en un periodo de 25 años, el 65\% de los individuos con 
antecedentes de consumo de carnes riesgosas estaban infectados, afectando mayoritariamente a zonas rurales, en invierno y primavera, por el incremento del consumo de carnes de cerdo y derivados $^{(17)}$. En Argentina se han desarrollado modelos predictivos de contraer Triquinelosis, dependiendo de las condiciones de procesamiento y consumo de carnes de cerdos, que señalan un riesgo de enfermar de 4,88 × $10^{-6}$, equivalente a 109 casos anuales ${ }^{(16)}$. Entre los factores de riesgo comunes que exhiben las personas infectadas están: pertenecer a poblaciones que no aplican revisión veterinaria de los alimentos cárneos, analfabetismo, desempleo, pobreza, que viven en condiciones insalubres y crían cerdos de traspatio sin cumplimiento de normas de higiene ${ }^{(18)}$.

El Echinococcus granulosus es el principal responsable de la hidatidosis en humanos, en su forma de Equinococosis quística (EQ) y afecta al 95\% de los 2-3 millones de personas que se estima padecen la enfermedad ${ }^{(6)}$. Entre los factores de riesgo de enfermar están: bajo nivel socioeconómico, escasa educación sanitaria, consumo de alimentos de procedencia animal, vivir en áreas rurales y convivir con perros que están en contacto con ganado o despojos de animales ${ }^{(8,9,15)}$. El parásito tiene como reservorio a caninos domésticos (E. granulosus) y salvajes (E. multilocularis), que albergan un parásito adulto que elimina huevos a través de sus fecas, que son ingeridos accidentalmente por otros animales que desarrollarán la forma quística en hígado y pulmón, conocido como $\mathrm{EQ}^{(19)}$. Los ovinos son los más afectados y el ser humano se infecta accidentalmente a través de manos, alimentos o agua contaminada ${ }^{(20)}$. El problema se presenta especialmente en las comunidades rurales donde están presentes los factores de riesgo, porque se encuentran las poblaciones de más bajos ingresos y menor educación, sumado a una tenencia de perros sin control de helmintiasis, características todas que sustentan la transmisión activa del parásito ${ }^{(6,20)}$.

El Hantavirus, o Virus Andes (ANDV) es endémico en Chile, Argentina y Brasil, único documentado de transmisión persona a persona y nosocomial $^{(21)}$. El riesgo de transmisión se asocia a presencia de roedores silvestres, Oligorzomys longicaudatus (ratón colilargo), único reservorio del virus $\mathrm{ANDV}^{(22)}$. La transmisión ocurre por inhalación de partículas víricas que han quedado expuestas a partir de orina, fecas y saliva de roedores reservorios ${ }^{(23)}$. Las zonas agrícolas, rurales y de deforestación son las de mayor riesgo de exposición ${ }^{(24)}$. La alteración del entorno y densa vegetación o ingreso a viviendas y galpones en mal estado favorecen el contagio y los grupos más expuestos son los trabajadores agrícolas ${ }^{(25)}$. En Chile la incidencia de Hantavirosis se presenta en la zona centro-sur del país, difiere según localización, pero es superior en el extremo sur, debido a la mayor densidad de población rural ${ }^{(21)}$.

Respecto al nivel de conocimientos y grupos de riesgo de estas enfermedades zoonóticas, en Argentina se reporta que trabajadores rurales reconocen la vía de transmisión de la Triquinelosis en comparación con la EQ, atribuido, entre otros aspectos, a que esta última es una enfermedad de lenta progresión y de ciclo complejo ${ }^{(26)}$. En Colombia se ha evidenciado que la Triquinelosis es considerada una infección de menor importancia respecto del Hanta u otras zoonosis y los grupos de mayor riesgo son las personas no profesionales y quienes desconocen factores de riesgos $^{(27)}$. Por su parte, en Nigeria, en general, son los cazadores de un sector rural los que tienen mayor riesgo de contagio de enfermedades zoonóticas en comparación a los comerciantes de zonas urbanas $^{(28)}$ y en Brasil el riesgo de adquirir zoonosis asociado a hábitos de la población, se enfoca a otras infecciones zoonóticas como la toxoplasmosis y leptospirosis ${ }^{(29)}$.

En Chile, el Ministerio de Salud considera a estas tres infecciones en el grupo de zoonosis de notificación obligatoria ${ }^{(11)}$ y a pesar de su gravedad a nivel país son escasos los estudios que relacionan el nivel de conocimientos con hábitos y costumbres asociadas a la transmisión de estas zoonosis $^{(30)}$.

La Región de la Araucanía, constituida por las provincias de Malleco y Cautín, está considerada entre las regiones más pobres de nuestro país ${ }^{(31)}$. En ella se encuentran localidades cordilleranas que reportan exposición a ganado bovino, caprino, ovino y en menor grado a cerdos. Curacautín 
distante a $86 \mathrm{~km}$ al noreste de la capital regional Temuco, Melipeuco a $95 \mathrm{~km}$ en dirección sureste y Lonquimay a $156 \mathrm{~km}$ al noreste, reúnen condiciones favorables para la transmisión de zoonosis $^{(32-35)}$ (Tabla 1), por su alto nivel de pobreza, baja escolaridad, con una población, particularmente en la ciudad de Angol, que tiene un promedio de años de estudio inferior al promedio de la región y el país.

Tabla 1. Características sociodemográficas generales de la población Región de la Araucanía.

\begin{tabular}{lccccc}
\hline Datos sociodemográficos & País & Región & Curacautín & Lonquimay & Melipeuco \\
\hline Nivel escolaridad (años)* $^{*}$ & 10,38 & 9,15 & 8,49 & 7,74 & 7,58 \\
Nivel pobreza \% (P. indigente/P. no indigente) & 15 & 27 & 34 & 31 & 24 \\
Etnia \% (mapuche) $^{*}$ & 6 & 30 & 13 & 39 & 40 \\
Ruralidad\% ** & & & 26,8 & 66,4 & 58,5 \\
Número de cabezas de ganado & & & & \\
Bovinos & & 26.464 & 23.897 & 11.277 \\
Caprinos & & 593 & 33.090 & 332 \\
Cerdos & & & 788 & 1.213 & 972 \\
Jabalies & & & 0 & 0 & 0 \\
Ovinos & & & & & \\
\hline
\end{tabular}

${ }^{*}$ Reportes estadísticos y comunales $2015^{(32-34)}$

${ }^{* *}$ Instituto Nacional de Estadísticas ${ }^{(35)}$

Durante el periodo 2000-2010 no se reportaron casos de Triquinelosis en estas comunas y las tasas de incidencia en este periodo (casos/10.000 habitantes) de EQ fue de 1,84 en Melipeuco; 1,32 en Curacautín y entre 1,73-7,79 en Lonquimay. Los casos de Hanta en el periodo alcanzaron hasta 1,84 en Melipeuco; 1,32 en Curacautín y 0,86 en Lonquimay ${ }^{(36)}$.

De manera de entender de mejor forma los patrones conductuales que predisponen a las patologías a explorar en este estudio es que se ha considerado atingente incluir el modelo de promoción de la Salud (MPS) de Pender como una referencia teórica, ya que éste permite identificar aquellos factores cognitivos-preceptuales que dependen de las características del ambiente en el que se insertan y existen, favoreciendo así las posible intervenciones preventivas específicas, en tanto conocimientos y prácticas; así como el fomento de la promoción de comportamientos saludables coherentes con el entorno en el que se desenvuelven los individuos ${ }^{(37)}$.

Considerando estos antecedentes, este trabajo tuvo como objetivo asociar el nivel de conocimiento y prácticas (hábitos y costumbres) responsables de la transmisión de Triquinelosis, Síndrome Cardiopulmonar por Hantavirus y Equinococosis quística (Hidatidosis), en habitantes de los sectores rurales de Curacautín, Lonquimay y Melipeuco.

\section{MATERIAL Y MÉTODO}

Tipo de estudio: Estudio descriptivo correlacional de corte transversal.

Unidad de análisis: adultos de ambos sexos, pertenecientes a comunidades rurales cordilleranas de 3 zonas de la Región de la Araucanía, inscritos y validados en las postas de salud locales de 
Icalma (Lonquimay), Santa Julia (Curacautín) y Santa María de Llaima (Melipeuco).

Muestra: La población de estudio se distribuyó, en una primera instancia a propósito de las unidades operativas comunales que participan del Programa de Desarrollo Local (PRODESAL), facilitando el Instituto de Desarrollo Agropecuario (INDAP) Araucanía el acceso a estos grupos y la cifra de individuos con los que trabajan: Melipeuco, 5.823 individuos; Lonquimay $1.214 \mathrm{y} \mathrm{Cu}$ racautín 2.350. Como referencia, el total de usuarios de 15 y más años en las tres postas rurales fue de 2.051 personas. Considerando la dificultad para acceder a los individuos en el contexto de la atención rural se propuso una muestra aleatoria simple para cada una de las localidades con 50 casos en cada una de ellas. La muestra a nivel general se definió en 150 casos, con un margen de error muestral máximo de $2,7 \%$. Para las 3 submuestras se definió un error máximo de \pm $4,7 \%$, con nivel de confianza del $95 \%$, estimando la proporción de la muestra en 3,0\%. En consecuencia, se logró aplicar el instrumento a un total de 149 personas, quienes eran los usuarios presentes en las postas durante las visitas del equipo, que consintieron informadamente a participar en dos ocasiones durante los meses de diciembre de 2013 y enero de 2014. Los criterios de inclusión fueron: mayores de 15 años, de ambos sexos, alfabetos y residentes en las áreas mencionadas. A través del Comité Ético Científico de la Universidad de La Frontera se consideró, además, una relación favorable de riesgo-beneficio y el respeto a los sujetos inscritos. Por tratarse de un estudio enfocado a pesquisar prácticas de fomento a la salud, se excluyeron individuos diagnosticados o que estuvieran cursando una de estas enfermedades o hayan tenido familiar cercano diagnosticado, en tratamiento, recuperado o fallecido por causa de alguna de estas zoonosis.

Recolección de datos: En una primera etapa se levantó información a través de Focal Group con líderes de la comunidad. A partir de esos resultados se diseñó una encuesta (Anexo), que fue validada por el ex director del laboratorio de inmu- noparasitología molecular de la Universidad de La Frontera, el Dr. Carlos Oberg Aravena, quien sugirió modificaciones en cuanto a la pertinencia de contenidos del área de la parasitología. La encuesta final fue construida con 2 dimensiones, uno que mide conocimiento y otros hábitos/costumbres (práctica) para cada una de las tres zoonosis. Cada ítem tiene una escala dicotómica que asigna la valoración 1 para la respuesta correcta y 0 para la respuesta incorrecta o sin responder. Por tratarse de población con bajo nivel de comprensión lectora se aplicó la encuesta a cada participante por encuestadores previamente capacitados, utilizando lenguaje coloquial para lograr una mejor comprensión de las preguntas, en una sala facilitada en las mismas postas de salud rural. Los resultados se expresaron en promedios de los porcentajes según las respuestas para cada dimensión.

La variable conocimiento se definió como el nivel de información que tiene la población sobre las características biológicas de las zoonosis (Triquinelosis, Hantavirus y Equinococosis quística), su transmisión, factores de riesgo y medidas de prevención demostrables. En la variable prácticas se consideraron conductas que tiene la población sobre hábitos y costumbres adquiridos según su cultura y su entorno, que podrían facilitar la transmisión de estas zoonosis.

Análisis de datos: El análisis de los datos se realizó en software $\mathrm{R}$ versión 3.0.2 FrisbeeSailing Copyright $\odot$ 2013. Se usaron pruebas bivariadas paramétricas según $r$ de Pearson y Chi cuadrado para medir la validez estadística de las preguntas aplicadas en el instrumento. Se aplicó un intervalo de confianza de $95 \%$.

\section{RESULTADOS}

Características sociodemográficas de los participantes: El grupo alcanzó un rango de edad de 1580 años, Curacautín con rango de 15 a 80 años, Lonquimay 17 a 71 años y Melipeuco 15-79 años. De estos el $57,1 \%$ eran mujeres y $42,9 \%$ varones, 
Curacautín y Melipeuco predominantemente femenina (23,5\% cada una) en comparación a Lonquimay, en donde el predominio fue masculino $(22,8 \%)$ (Tabla 2$)$.

Nivel de conocimientos y prácticas para cada una de las zoonosis por comuna (Tabla 3): De las 27 preguntas analizadas 25 resultaron estadísticamente significativas $(\mathrm{p}<0,05)$. La población total reporta un $65 \%$ de conocimiento correcto en Triquinelosis, 73\% en Hanta y 39\% en Equinococosis Quística (EQ). Respecto al conocimiento específico del reservorio de estas infecciones, los participantes reconocen correctamente el reservorio para Hanta y Triquinelosis, no así para EQ. Respecto a la transmisión de la enfermedad, solo el 9\% de las respuestas fue correcta, el 38\% atribuyó la transmisión de EQ a los perros y una gran mayoría a la inhalación de pelos de gato. En relación al riesgo de enfermar, el $88 \%$ de la población reconoció correctamente que el riesgo de contagio de Hanta se presenta al ingresar a construcciones cerradas sin ventilar y el $71 \%$ a la exposición a fluidos de los roedores. En Triquinelosis, el $64 \%$ de la población atribuye que el riesgo de enfermar es por el consumo de carne de cerdo cruda y el 76\% reconoce que la no realización del examen veterinario es una omisión que favorece la transmisión. Destaca que el $42 \%$ valida al jugo de limón como un líquido capaz de cocer la carne. En EQ el 60\% de los participantes reconoce que la falta de desparasitación de los perros favorece la transmisión y el 71\% reconoce dar vísceras a los perros aun cuando éstas estén infectadas con la forma quística del parásito. Un resultado de conocimiento que no fue estadísticamente significativo fue el que un $50 \%$ de los encuestados reconoce necesaria la desparasitación del perro para evitar EQ.

Tabla 2. Características sociodemográficas de los participantes.

\begin{tabular}{lcccc}
\hline Características & Curacautín & Lonquimay & Melipeuco & Total \\
\hline Población Localidad rural & 2350 & 1214 & 5823 & 9387 \\
Población adulta usuaria de las postas rurales & 319 & 332 & 1400 & 2051 \\
Población encuestada (n, \%) & $50(33,6)$ & $49(32,9)$ & $50(33,6)$ & $149(100)$ \\
Edad (Media, DE) & $50,32 \pm 19.67$ & $41,9 \pm 16,17$ & $50,4 \pm 18,61$ & $47,57 \pm 18,54$ \\
Mujeres (n, \%) & $35(23,5)$ & $15(10,1)$ & $35(23,5)$ & $85(57,1)$ \\
Hombres (n, \%) & $15(10,1)$ & $34(22,8)$ & $15(10,1)$ & $64(42,9)$ \\
\hline
\end{tabular}

Prácticas: El 70\% reporta prácticas correctas para Triquinelosis, el 96\% afirma consumir la carne de cerdo bien cocida, el $85 \%$ señala realizar el examen veterinario de las carnes y el $86 \%$ considera necesario mantener limpio el lugar donde se crían los cerdos. Aun así, el 33\% valida al jugo de limón como elemento útil para detectar la presencia de larvas en la carne de consumo. Respecto al Hanta, el $84 \%$ reporta correctas prácticas, de ellos, el $89 \%$ reconoce ventilar los lugares cerrados y el 64\% protege la vía aérea para el ingreso a estos lugares. Tan solo el $37 \%$ considera posibilidades de contagio aun cuando no ven roedo- res en su entorno y el 66\% reconoce que al vivir en una zona rural se expone a la transmisión. El $84 \%$ de la población reconoce que las lechuzas y culebras son depredadores del roedor y el 99\% señala mantener limpio y desmalezado el entorno de sus hogares. Acerca de EQ, el 84\% reporta prácticas correctas, representado por un $74 \%$ que afirma dejar lejos del alcance de los perros los restos de animales faenados, un $84 \%$ que reconoce desparasitar a los perros al menos 1 vez al año y un $94 \%$ que señala lavarse las manos después del contacto con perros. Un resultado de prácticas que no fue estadísticamente significativo fue 
Tabla 3. Nivel de conocimientos (C) y prácticas (P) asociadas a zoonosis (Respuestas correctas de los participantes).

\begin{tabular}{|c|c|c|c|c|c|c|c|c|c|c|c|c|c|}
\hline \multirow{2}{*}{$\begin{array}{c}\mathrm{N}^{\circ} \\
\text { pregunta }\end{array}$} & \multirow{2}{*}{ Zoonosis } & \multirow{2}{*}{ Preguntas } & \multirow{2}{*}{$\mathrm{C}$} & \multirow{2}{*}{$\mathrm{P}$} & \multicolumn{2}{|c|}{ Curacautín } & \multicolumn{2}{|c|}{ Lonquimay } & \multicolumn{2}{|c|}{ Melipeuco } & \multicolumn{2}{|c|}{ Total Pregunta } & \multirow{2}{*}{$\begin{array}{c}\text { p-value } \\
\mathrm{Chi}^{2}\end{array}$} \\
\hline & & & & & $\mathrm{n}$ & $\%$ & $\mathrm{n}$ & $\%$ & $\mathrm{n}$ & $\%$ & $\mathrm{n}$ & $\%$ & \\
\hline 1 & \multirow{9}{*}{ Triquinelosis } & ¿Qué animal me puede enfermar de triquinosis? & $\mathrm{x}$ & & 42 & 84 & 36 & 73 & 38 & 76 & 116 & 78 & 0,001 \\
\hline 2 & & El riesgo de enfermar de triquinosis ocurre cuando... & $\mathrm{x}$ & & 36 & 72 & 29 & 59 & 30 & 60 & 95 & 64 & 0,000 \\
\hline 3 & & $\begin{array}{l}\text { Si como carne de chancho sin hacerle el examen veterinario, tengo } \\
\text { riesgo de.... }\end{array}$ & $\mathrm{x}$ & & 44 & 88 & 31 & 63 & 39 & 78 & 114 & 76 & 0,001 \\
\hline 1a & & La carne de cerdo la como siempre bien cocida. & & $\mathrm{x}$ & 49 & 98 & 47 & 96 & 47 & 94 & 143 & 96 & 0,000 \\
\hline $2 \mathrm{a}$ & & $\begin{array}{l}\text { El lugar donde comen los chanchos, debe ser limpiado una vez a la } \\
\text { semana. }\end{array}$ & & $\mathrm{x}$ & 45 & 90 & 38 & 78 & 45 & 90 & 128 & 86 & 0,000 \\
\hline $3 \mathrm{a}$ & & $\begin{array}{l}\text { Siempre que faeno un chancho, le realizo el examen de la triquina } \\
\text { con el veterinario. }\end{array}$ & & $\mathrm{x}$ & 46 & 92 & 39 & 80 & 41 & 82 & 126 & 85 & 0,000 \\
\hline $4 a$ & & $\begin{array}{l}\text { Cuando consumo carne de lechones o chanchos nuevos no me } \\
\text { enfermo de triquina. }\end{array}$ & & $\mathrm{x}$ & 30 & 60 & 23 & 47 & 18 & 36 & 71 & 48 & 0,560 \\
\hline $5 \mathrm{a}$ & & El jugo de limón cuece la carne. & $\mathrm{x}$ & & 19 & 38 & 16 & 33 & 27 & 54 & 62 & 42 & 0,040 \\
\hline $6 \mathrm{a}$ & & $\begin{array}{l}\text { Con el jugo de limón puedo saber si la carne de chancho tiene } \\
\text { triquina. }\end{array}$ & & $\mathrm{x}$ & 24 & 48 & 8 & 16 & 18 & 36 & 50 & 33 & 0,000 \\
\hline 4 & \multirow{9}{*}{ Hanta } & ¿Qué animal me puede enfermar de Hantavirus? & $\mathrm{x}$ & & 49 & 98 & 43 & 88 & 44 & 88 & 136 & 91 & 0,000 \\
\hline 5 & & ¿Dónde hay más riesgo de encontrar Hantavirus? & $\mathrm{x}$ & & 45 & 90 & 39 & 80 & 47 & 94 & 131 & 88 & 0,000 \\
\hline 6 & & $\begin{array}{l}\text { Cuando los ratones ingresan a la casa o bodegas y orinan o defecan } \\
\text { en ella, me arriesgo a enfermar de... }\end{array}$ & $\mathrm{x}$ & & 45 & 90 & 25 & 51 & 36 & 72 & 106 & 71 & 0,000 \\
\hline $13 \mathrm{a}$ & & $\begin{array}{l}\text { Cuando ingreso a bodegas deshabitadas, ventilo mínimo } 30 \text { minutos } \\
\text { el lugar. }\end{array}$ & & $\mathrm{x}$ & 44 & 88 & 40 & 82 & 47 & 94 & 131 & 88 & 0,002 \\
\hline $14 \mathrm{a}$ & & $\begin{array}{l}\text { Cuando ingreso a bodegas cerradas, me cubro la nariz con pañuelos } \\
\text { o mascarilla. }\end{array}$ & & $\mathrm{x}$ & 33 & 66 & 27 & 55 & 35 & 70 & 95 & 64 & 0,000 \\
\hline $15 \mathrm{a}$ & & $\begin{array}{l}\text { Desmalezo y limpio el entorno de mi casa para mantener el lugar } \\
\text { libre de roedores. }\end{array}$ & & $\mathrm{x}$ & 50 & 100 & 48 & 98 & 50 & 100 & 148 & 99 & 0,000 \\
\hline $16 \mathrm{a}$ & & $\begin{array}{l}\text { Si no veo roedores o ratones alrededor de mi casa, no me contagio } \\
\text { de Hanta virus. }\end{array}$ & $\mathrm{x}$ & & 21 & 42 & 18 & 37 & 16 & 32 & 55 & 37 & 0,001 \\
\hline $17 \mathrm{a}$ & & Las culebras y lechuzas nos protegen del Hanta. & $\mathrm{x}$ & & 41 & 82 & 40 & 82 & 44 & 88 & 125 & 84 & 0,000 \\
\hline $18 \mathrm{a}$ & & Quienes vivimos en el campo, no nos enfermamos del Hanta. & $\mathrm{x}$ & & 36 & 72 & 30 & 61 & 32 & 64 & 98 & 66 & 0,000 \\
\hline 7 & \multirow{9}{*}{ Hidatidosis } & $\begin{array}{l}\text { ¿Qué puede ocurrir con la salud de mi familia si los perros no han } \\
\text { tomado antiparasitarios? }\end{array}$ & $\mathrm{x}$ & & 26 & 52 & 34 & 69 & 29 & 58 & 89 & 60 & 0,010 \\
\hline 8 & & $\begin{array}{l}\text { Tengo riesgo de enfermar de las bolsas de agua o bolas de pelo } \\
\text { (quistes del pulmón) cuando:... }\end{array}$ & $\mathrm{x}$ & & 25 & 50 & 24 & 49 & 26 & 52 & 75 & 50 & 0,930 \\
\hline 9 & & ¿Qué animal me puede enfermar de hidatidosis? & $\mathrm{x}$ & & 6 & 12 & 34 & 69 & 17 & 34 & 57 & 38 & 0,004 \\
\hline $7 \mathrm{a}$ & & $\begin{array}{l}\text { Cuando faeno animales, los restos no utilizados los boto en lugares } \\
\text { fuera del alcance de los perros. }\end{array}$ & & $\mathrm{x}$ & 34 & 68 & 35 & 71 & 41 & 82 & 110 & 74 & 0,000 \\
\hline $8 \mathrm{a}$ & & Luego de acariciar a mi perro me lavo las manos. & & $\mathrm{x}$ & 46 & 92 & 46 & 94 & 48 & 96 & 140 & 94 & 0,000 \\
\hline $9 \mathrm{a}$ & & A mi perro le doy la pastilla antiparasitaria una vez al año. & & $\mathrm{x}$ & 40 & 80 & 45 & 92 & 40 & 80 & 125 & 84 & 0,000 \\
\hline $10 \mathrm{a}$ & & Los quistes del pulmón salen al respirar o inhalar pelos de perro. & $\mathrm{x}$ & & 3 & 6 & 2 & 4 & 6 & 12 & 11 & 7 & 0,000 \\
\hline $11 \mathrm{a}$ & & Los gatos nos enferman de las bolas de pelo o bolsas de agua. & $\mathrm{x}$ & & 3 & 6 & 6 & 8 & 4 & 12 & 13 & 9 & 0,000 \\
\hline $12 \mathrm{a}$ & & $\begin{array}{l}\text { Cuando la pana de la oveja sale con bolsas de agua, se la doy al } \\
\text { perro. }\end{array}$ & $\mathrm{x}$ & & 36 & 72 & 38 & 78 & 32 & 64 & 106 & 71 & 0,000 \\
\hline
\end{tabular}

Lenguaje ajustado al nivel sociocultural de la población en estudio: Bolsas de agua o bolas de pelo= Equinococosis quistica o Quiste hidatídico; Chancho=cerdo; Pana=hígado o vísceras; Triquinosis o triquina=triquinelosis. $\mathrm{C}=$ Conocimiento, $\mathrm{P}=$ Prácticas. (Significancia estadística: -value $<0,05$ )

que un $48 \%$ reconoce al lechón como animal sin riesgo de contagiar Triquinelosis.

Asociación entre conocimiento y prácticas ( $\mathrm{Fi}$ gura 1): Al relacionar estas dimensiones se evidencian buenas prácticas para las tres zoonosis (Figura 1a), aun cuando el conocimiento es inferior, principalmente en EQ y sin asociación entre ambas dimensiones $\left(r^{2}=0,117\right)$. En la Figura $1 b$ se observa asociación proporcional entre ambas dimensiones para Triquinelosis, con tendencia lineal positiva $\left(r^{2}=0,78\right)$ y es manifiesto que las correctas prácticas son frecuentes. En Lonquimay se aprecia la menor relación entre conocimiento y práctica y en Curacautín mejor relación entre ellas. En el Hanta, la asociación entre las dimensiones (Figura 1c) muestra que el nivel de conocimiento es proporcional con adecuadas prácticas. Como dato atípico se observa a la localidad de Melipeuco con las mejores prácticas, aun cuando los participantes de esta localidad muestran un nivel de conocimiento intermedio. En Lonquimay se aprecia relación proporcional entre conocimiento y prácticas, aun cuando pre- 
senta los valores más bajos $\left(r^{2}=0,50\right)$. La relación entre conocimiento y prácticas en EQ (Figura 1d) es proporcional entre ambas dimensiones $\left(r^{2}=0,70\right)$ y destaca como la zoonosis de la que se tiene menor conocimiento. Se observa un importante nivel de desinformación pues ninguna de las 3 localidades logra valores superiores al 50\%. Curacautín es la localidad en donde se reporta el menor valor en ambas dimensiones, Melipeuco con las mejores prácticas y nivel de conocimiento medio y Lonquimay con mejor nivel de conocimiento, a pesar de presentar prácticas medias.

Figura 1. Relación de conocimiento y prácticas asociado a transmisión de zoonosis.
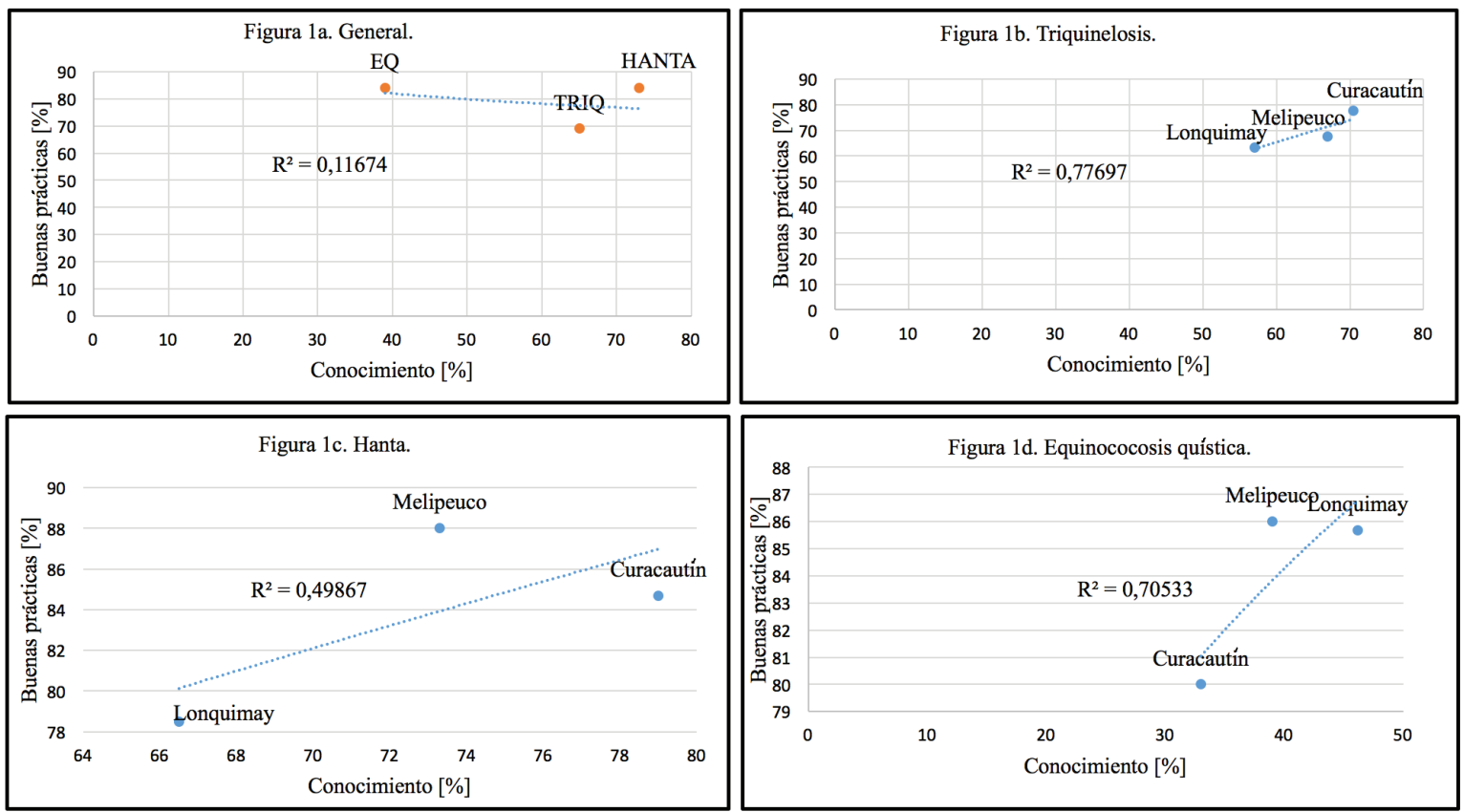

Figura 1a. Relación entre conocimientos y prácticas para las 3 zoonosis de acuerdo a localidad; Figura 1b. Relación entre conocimientos y prácticas asociadas a la transmisión de triquinelosis; Figura 1c. Relación entre conocimientos y prácticas asociadas con la transmisión de Hanta; Figura 1d. Relación entre conocimientos y prácticas asociadas con la transmisión de Equinococosis quística. (Pruebas de correlación paramétricas del coeficiente $r$ de acuerdo a Pearson).

\section{DISCUSIÓN}

Respecto a Triquinelosis, se aprecian resultados similares en ambas dimensiones, sin embargo, hay mejores prácticas que conocimiento. Aunque se ha reportado incremento de casos de Triquinelosis en la población mundial ${ }^{(38)}$, esto no se refleja a nivel local, lo que podría atribuirse al bajo número de porcinos que se crían en estas localidades, según reportes estadísticos comunales ${ }^{(34-36)}$. $\mathrm{Al}$ revisar el conocimiento reportado de Triquinosis, éste alcanza un nivel aceptable y se le ubica en el segundo lugar, similar a otros estudios ${ }^{(26)}$ que indican más conocimiento sobre el agente y transmisión en Triquinelosis y menos respecto a EQ. De prácticas de Triquinelosis, la población reconoce que consumir la carne de cerdo cocida y mantener adecuada crianza de los cerdos es un buen hábito preventivo. Si bien la población manifiesta no conocer con claridad qué enfermedad se evita al realizar el examen del cerdo, sí considera necesario realizarlo para evitar enfermar. Destaca que las personas aún validan al jugo de limón como una sustancia capaz de cocer la carne y de detectar las larvas presentes en la carne infectada, explicable, tal vez, por las características sociodemográficas de la población establecidas 
por Singh et al. ${ }^{(39)}$, cuando señala que estas malas prácticas se pueden atribuir a diversas causas, tales como las condiciones de vida, el difícil acceso a centros veterinarios, el costo del examen que encarece el sistema de vida, etc., todas situaciones que obligan a las personas a recurrir a otros procedimientos para detectar carnes infectadas. Estas condiciones, según Franssen ${ }^{(40)}$ aumentan el riesgo de transmisión que, además, se ampara en la falta de inspección veterinaria de las carnes de cerdo y todas estas malas prácticas también se asocian al bajo nivel educacional y económico de la población. Contrario a lo que señala la literatura $^{(30)}$ en donde se indica que las malas prácticas de crianza de cerdos están presentes en poblaciones rurales, se destaca en este estudio el hallazgo de la crianza de cerdos en lugares limpios. Aun cuando en la última década no se reportan casos de Triquinelosis en estas localidades, se requiere estar siempre alerta a los posibles brotes debido a los malos hábitos y costumbres que aún permanecen en la población ${ }^{(17)}$. Se debe seguir fomentando una crianza de cerdos en lugares seguros y un consumo de carne adecuadamente cocida ${ }^{(1)}$.

Respecto del Hanta, es la zoonosis más conocida entre la población rural, al igual que en los estudios de Cediel et al. ${ }^{(27)}$. Se evidencia buen nivel de conocimientos y prácticas, la gran mayoría reconoce al roedor como agente responsable de la transmisión, identifican mayor riesgo de contagio al ingresar a lugares cerrados y deshabitados. Es importante considerar que ellos reconocen menor riesgo de contagio si no ven roedores y subestiman la transmisión por contacto con fluidos del reservorio, aun cuando éstos son fuente de transmisión del virus ${ }^{(41)}$. La población valora más ventilar los lugares que han estado deshabitados que el cubrir la vía aérea, quedando igualmente expuestos al ingreso del virus por esta vía. Destaca que la población subestima el riesgo de contagio por ser residentes de zonas rurales, contrario a los reportes de Pinto et al. ${ }^{(24)}$, en donde la población percibía que vivir en el área rural era un factor de riesgo de contagio. Si hay factores protectores como reconocer a los depredadores y mantener el entorno limpio y desmalezado, se favorece el control del reservorio.
Se puede precisar que la población está mejor preparada para evitar el contagio de Hanta que para las otras zoonosis, aunque se mantienen algunas prácticas que exponen a la transmisión ${ }^{(14)}$. Esto explicaría la permanencia de casos presentes en estas localidades. Aunque actualmente la autoridad sanitaria desarrolla campañas dirigidas a educar a la comunidad, que podría explicar el adecuado nivel de conocimiento encontrado en este estudio, estas medidas no alcanzan a provocar cambios en los hábitos o costumbres y las medidas de control no se aplican en su totalidad, sumado a que también hay factores sociodemográficos asociados ${ }^{(25)}$.

Respecto a Equinococosis quística, es preocupante el nivel de desinformación en la población, condición favorable para la permanencia de la parasitosis ${ }^{(8,15)}$. Esto podría explicarse por lo complejo del ciclo vital del parásito, la lenta evolución clínica de la enfermedad y su difícil nombre científico, que en suma dificultan el conocimiento ${ }^{(21)}$. Se destaca que el entorno donde vive la población asociado a la crianza de ganado bovino, ovino y caprino, facilita la transmisión hacia los perros ${ }^{(9)}$. Respecto a la valoración de la desparasitación de los perros, se corroboran los reportes de Cediel et $\mathrm{al}^{(27)}$, que reconocen a esta práctica como un elemento protector, aun cuando no reconocen al perro como hospedero responsable de la transmisión. A pesar de validar la desparasitación, la frecuencia del tratamiento es insuficiente para mantener controlado al parásito, y esa situación aumenta el riesgo de contagio ${ }^{(42)}$. La transmisión también se ve favorecida por la falta de conocimiento del mecanismo de transmisión del parásito, asociando la inhalación de pelos de gato y en menor grado la presencia de huevos del parásito eliminados en las fecas de los perros ${ }^{(11)}$. Un importante grupo de la población reconoce que no se deben dar desperdicios de animales faenados a los perros, pero esto se contradice con la práctica de dar las vísceras infectadas a los perros pues con ello se facilita la continuidad del desarrollo del ciclo parasitario ${ }^{(20)}$. Estos factores, sumado a la falta de desparasitación de los perros, contamina el ambiente con los huevos del parásito y deja expuesta a la población y al ganado, al con- 
tagio y desarrollo de las formas larvales ${ }^{(7,43)}$. Lo anterior se refleja en la alta incidencia de EQ en estas zonas geográficas. Sorprende que la población señala buena adherencia al lavado de manos después del contacto con perros, contrario a los reportes de Venegas et al. ${ }^{(11)}$. En tanto a los resultados de EQ y Triquinelosis que no resultaron estadísticamente significativos es posible argüir que son probablemente azarosos y de repetir su aplicación se sugiere hacerlo con una muestra más grande.

\section{CONCLUSIONES}

Según los antecedentes, es posible señalar que la población total posee más conocimientos de Hanta y Triquinelosis que de EQ. De las prácticas, se reportan hábitos y costumbres más favorables para Hanta y EQ que para Triquinelosis. Del conocimiento y prácticas específicas, se aprecia que la mayoría de la población reconoce al reservorio de Triquinella spiralis y Hantavirus, siendo mejor reconocido para este último y no así para Echinococcus granulosus.

Es posible confirmar que las condiciones de vida de estos grupos poblacionales; ruralidad, difícil acceso a centros urbanos, tenencia de perros y crianza de ganado, bajo nivel económico y de educación, entre otros, dificultan el conocimiento más que las buenas prácticas aplicadas a nivel local. Lo anterior condicionó las limitantes del estudio, entre las que destaca el difícil acceso a estos sectores, lo que de alguna forma circunscribe el tamaño de la muestra y condiciona la recolección de datos, además de los posibles sesgos propios del uso de datos autoinformados.

Muchas de las buenas prácticas ocurren por conductas adquiridas, sin embargo, el desconocimiento de bases teóricas sólidas expone a la población, por lo cual se sugiere que expertos en el tema, veterinarios, trabajadores de salud, técnicos agrícolas y otros involucrados sociales, coordinen y desarrollen intervenciones dirigidas a los grupos más vulnerables, en forma sistemática e interdisciplinaria, considerando condiciones específicas de la población para reforzar las buenas prácticas y evitar conductas riesgosas.

Es necesario destacar que Curacautín es la localidad con menor conocimientos y prácticas de EQ, sin embargo, presenta inferior reporte de casos. A su vez, Lonquimay es la localidad más alejada de centros urbanos, con mayor prevalencia de EQ, aun cuando el conocimiento y prácticas alcanza valores bajos, ésta es la localidad mejor evaluada de las tres. Atendiendo a estos antecedentes, es necesario centrar las medidas de control de EQ en el manejo responsable de los perros, alimentación y desparasitación periódica, sin subestimar el Hanta y la Triquinelosis.

Considerando los resultados desde la perspectiva del Modelo de Promoción de la Salud de Pender, es posible entender el que mayor conocimiento no sea necesariamente el origen de mejores prácticas o que las prácticas saludables no estén irremediablemente condicionadas por el nivel de conocimientos de los individuos. Teniendo en cuenta lo anterior es posible utilizar el MPS para valorar la pertinencia de las intervenciones de Enfermería, siendo un importante marco referencial para comprender y promover las actitudes, motivaciones y acciones de las personas desde la orientación de la promoción de la salud como elemento fundamental del cuidado de Enfermería.

Agradecimientos: Agradecemos el apoyo de quienes facilitaron el acceso a la población participante del estudio para el levantamiento de la información: Verónica Abarzúa, Enfermera, Departamento de Salud, Municipalidad de $\mathrm{Cu}$ racautín; Alejandra A. Cáceres G., Encargada de Proder, Curacautín; José Quemener M., Jefe Técnico, PRODESAL, Lonquimay; Pía Paz Díaz, Jefe Técnico, PRODESAL, Lonquimay; Pedro Cortés, Matrón, Departamento de Salud, Municipalidad de Lonquimay; Luis Mondaca R., Profesional Técnico Agrícola, PRODESAL, Melipeuco; María A. Romero S., Ingeniero Agrónoma, PRODESAL, Melipeuco. 


\section{REFERENCIAS}

1. Murrell KD. Zoonotic foodborne parasites and their surveillance. Rev Sci Tech. 2013; 32(2): 559-569.

2. CDC: Centers for Disease Control and Prevention. [Internet]. Atlanta: CDC; 2012 [cited 5 Apr 2016]. Available from: https://www. cdc.gov/parasites/trichinellosis/epi.html

3. Ng-Nguyen D, Stevenson MA, Traub RJ. A systematic review of taeniasis, cisticercosis and trichinellosis in Vietnam. Parasit Vectors [Internet]. 2017 Mar [cited 2018 Oct 25]; 10(1): 150. Available from: https://www.ncbi. nlm.nih.gov/pubmed/28320455

4. Van De N, Thi Nga V, Dorny P, Vu Trung N, Ngoc Minh P, Trung Dung D et al. Trichinellosis in Vietnam. Am J Trop Med Hyg [Internet]. 2015 Jun [cited 2018 Dec 3]; 92(6): 1275-70. Available from: https://www.ncbi. nlm.nih.gov/pubmed/25846295

5. Instituto de Salud Pública de Chile. Resultados de diagnóstico y confirmación de laboratorio Triquinosis. Chile, 2005-2015 [Internet]. Vol. 6. Santiago, Chile: ISP; 2016. [citado 25 Oct 2018]. 9 p. Disponible en: http:// www.ispch.cl/content/23173

6. Armiñanzas C, Gutiérrez M, Fariñas C. Hidatidosis: aspectos epidemiológicos, clínicos, diagnósticos y terapéuticos. Rev Esp Quimioter [Internet]. 2015 Jun [citado 25 Oct 2018]; 28(3): 116-124. Disponible en: http://seq.es/ seq/0214-3429/28/3/farinas.pdf

7. Afonso E, Knapp J, Tête N, Umhang G, Rieffel D, Van Kesteren F et al. Echinococcus multilocularis in Kyrgyzstan: similarity in the Asian EmsB genotypic profiles from village populations of Eastern mole voles (Ellobius tancrei) and dogs in the Alay valley. J Helminthol [Internet]. $2015 \mathrm{Nov}$ [cited 2018 Oct 25]; 89(06): 664-670. Available from: http:// www.ncbi.nlm.nih.gov/pubmed/26137938

8. Liu C-N, Xu Y-Y, Cadavid-Restrepo AM, Lou Z-Z, Yan H-B, Bao-Quan Fu L et al. Estimating the prevalence of Echinococcus in domestic dogs in highly endemic for echino- coccosis. Infect Dis Poverty [Internet]. 2018 Dec [cited 2018 Oct 25]; 7:77. Available from: https://www.ncbi.nlm.nih.gov/pmc/articles/ PMC6083587/

9. Abdybekova A, Sultanov A, Karatayev B, Zhumabayeva A, Shapiyeva Z, Yeshmuratov $\mathrm{T}$ et al. Epidemiology of echinococcosis in Kazakhstan: an update. J Helminthol [Internet]. 2015 Nov [cited 2018 Oct 25]; 89(6): 647-650. Available from: https://www.ncbi. nlm.nih.gov/pubmed/26160276

10. Acosta-Jamett G, Vargas R, Ernst S. Caracterización epidemiológica de hidatidosis humana y animal en la Región de Los Ríos, 1999-2009. Rev Chilena Infectol [Internet]. 2016 Ago [citado 25 Oct 2018]; 33(4): 419427. Disponible en: https://scielo.conicyt.cl/ pdf/rci/v33n4/art06.pdf

11. Venegas J, Espinoza S, Sánchez G. Estimación del impacto económico de la equinococosis quística en Chile y análisis de las posibles causas que han dificultado su erradicación. Rev Med Chil [Internet]. 2014 Ago [citado 25 Oct 2018]; 142(8): 1023-1033. Disponible en: https://www.ncbi.nlm.nih.gov/pub$\mathrm{med} / 25424675$

12. Vial C, Martínez-Valdebenito C, Ríos S, Martínez J, Vial PA, Ferres M et al. Molecular method for the detection of Andes Hantavirus infection: validation for clinical diagnostics. Diagn Microbiol Infect Dis [Internet]. 2016 Jan [cited 2018 Oct 25]; 84(1): 36-39. Available from: http://www.ncbi.nlm.nih. gov/pubmed/26508102

13. Juan EE, Provensal MC, Steinmann AR. Space Use and Social Mating System of the Hantavirus Host, Oligoryzomys longicaudatus. Ecohealth [Internet]. 2018 Mar [cited 2018 Oct 25]; 15(1): 96-108. Available from: http:// www.ncbi.nlm.nih.gov/pubmed/29196828

14. Vallejos-Espíndola JA, Troncoso-González MA. Impacto medioambiental en la incidencia del Síndrome Cardiopulmonar por Hantavirus en Chile. Rev Med Chile. [Internet]. 2014 Abr [citado 25 Oct 2018]; 142(4): 538-539. Disponible en: https://scielo.conicyt.cl/scielo.php?script=sci_arttext\&pi- 
$\mathrm{d}=$ S0034-98872014000400021

15. Barnes AN, Davaasuren A, Baasandagva U, Gray GC. A systematic review of zoonotic enteric parasitic diseases among nomadic and pastoral people. PLoS One [Internet]. 2017 Nov. [cited 2018 Oct 25]; 12(11): e0188809. Available from: https:/www.ncbi.nlm.nih. gov/pubmed/29190664

16. Sequeira GJ, Zbrun MV, Soto LP, Astesana DM, Blajman JE, Rosmini MR et al. Quantitative Risk Assessment of Human Trichinellosis Caused by Consumption of Pork Meat Sausages in Argentina. Zoonoses Public Health [Internet]. 2016 Mar [cited 2018 Oct 25]; 63(2): 167-176. Available from: https:// www.ncbi.nlm.nih.gov/pubmed/26227185

17. Dobrescu C, Hriscu H, Emandi M, Zamfir C, Nemet C. Consumption of untested porkcontributed to over two-thousand clinical cases of human trichinellosis in Romania. Folia Parasitol (Praha) [Internet]. 2014 Dec [cited 2018 Oct 25]; 61(6): 558-560. Available from: https:/www.ncbi.nlm.nih.gov/ pubmed/25651698

18. Rostami A, Gamble HR, Dupouy-Camet J, Khazan H, Bruschi F. Meat sources of infection for outbreaks of human trichinellosis. Food Microbiol [Internet]. 2017 Jun. [cited 2018 Oct 25]; 64: 65-71. Available from: https://www.ncbi.nlm.nih.gov/ pubmed/28213036

19. Çiçekli Ö, Akgül T. An unusual cause of mass localized on vastus lateralis muscle in childhood: Hydatid cyst. Int J Surg Case Rep [Internet]. 2015 [cited 2018 Oct 25]; 6: 179-181. Available from: https:/www.ncbi.nlm.nih. gov/pubmed/25544487

20. Pakala T, Molina M, Wu GY. Hepatic Echinococcal Cysts: A Review. J Clin Transl Hepatol. 2016; 4(1): 39-46.

21. Martínez-Valdebenito C, Calvo M, Vial C, Mansilla R, Marco C, Palma RE et al. Person-to-person household and nosocomial transmission of Andes Hantavirus, Southern Chile, 2011. Emerg Infect Dis [Internet]. 2014 Oct [cited 2018 Oct 25]; 20(10): 16371644. Available from: http://www.ncbi.nlm. nih.gov/pubmed/25272189

22. Muñoz-Pedreros A, Gil C, Yáñez J, Rau JR, Möller P. Trophic ecology of two raptors, Barn Owl (Tyto alba) and White-tailed Kite (Elanus leucurus), and possible implications for biological control of Hantavirus reservoir in Chile. Wilson J Ornithol [Internet]. 2016 Jun [cited 2018 Oct 25]; 128(2): 391403. Available from: http://www.bioone.org/ doi/10.1676/wils-128-02-391-403.1

23. Ermonval M, Baychelier F, Tordo N. What Do We Know about How Hantaviruses Interact with Their Different Hosts? Viruses [Internet]. 2016 Aug [cited 2018 Oct 25]; 8(8). Available from: http://www.ncbi.nlm.nih. gov/pubmed/27529272

24. Pinto Junior VL, Hamidad AM, Albuquerque Filho D de O, Dos Santos VM. Twenty years of Hantavirus pulmonary syndrome in Brazil: a review of epidemiological and clinical aspects. J Infect Dev Ctries [Internet] 2014 Feb [cited 2018 Oct 25]; 8(2): 137-142. Available from: https://www.ncbi.nlm.nih.gov/ pubmed/24518622

25. Talmon G, Herrero E, Arezo M, Cantoni G, Larrieu E. Condiciones para la transmisión de Hantavirus en Zona Andina de Río Negro, Argentina. Medicina (B Aires) [Internet]. 2014 Ago [citado 25 Oct 2018]; 74(5): 378384. Disponible en: https://www.ncbi.nlm. nih.gov/pubmed/25347899

26. Molineri AI, Signorini ML, Tarabla HD. Conocimiento de las vías de transmisión de laszoonosis y de las especies afectadas entre los trabajadores rurales. Rev Argent Microbiol [Internet]. 2014 Mar [citado 25 Oct 2018]; 46(1): 7-13. Disponible en: http://www.scielo.org.ar/scielo.php?script=sci_arttext\&pi$\mathrm{d}=$ S0325-75412014000100003

27. Cediel N, Villamil LC, Romero J, Rentería L, De Meneghi D. Setting priorities for surveillance, prevention, and control of zoonoses in Bogotá, Colombia. Rev Panam Salud Publica [Internet]. 2013 May [cited 2018 Oct 25]; 33(5): 316-324. Available from: https:// www.ncbi.nlm.nih.gov/pubmed/23764662

28. Ozioko KU, Okoye CI, Obiezue RN, Agbu 
RA. Knowledge, attitudes, and behavioural risk factors regarding zoonotic infections among bushmeat hunters and traders in Nsukka, southeast Nigeria. Epidemiol Health [Internet]. 2018 Jun [cited 2018 Oct 25]; 40: e2018025. Available from: http://www.ncbi. nlm.nih.gov/pubmed/29909609

29. Fonseca TC, Sousa FF, Carballo FP, Fonseca AR, da Silva Rabelo DMR. Fatores associados às enteroparasitoses em crianças usuárias de creches comunitárias. Ciência \& Saúde [Internet]. 2017 Out [citado 2018 Out 26]; 11(1): 33-40. Disponível em: http://revistaseletronicas.pucrs.br/ojs/index.php/faenfi/ article/view/27909

30. Lisboa-Navarro R, González J, Junod T, Melín-Coloma M, Landaeta-Aqueveque C. Conocimientos y prácticas sobre hidatidosis y triquinosis en usuarios y acompañantes del Hospital Comunitario Salud Familiar El Carmen, Región del Biobío, Chile. Rev Chilena Infectol. [Internet] 2016 Ago [citado 25 Oct 2018]; 33(4): 474-476. Disponible en: https:// scielo.conicyt.cl/scielo.php?script=sci_arttext\&pid=S0716-10182016000400016

31. Ministerio de Desarrollo Social. Encuesta de Caracterización Socioeconómica Nacional (CASEN). Indicadores de Pobreza. Santiago, Chile: Ministerio de Desarrollo Social; 2017.

32. Biblioteca del Congreso Nacional de Chile/ BCN. Reportes Estadísticos Comunales 2015 Lonquimay [Internet]. Valparaíso: BCN; 2015 [citado 3 Dic 2018]. [aprox. 36 pantallas]. Disponible en: https://reportescomunales.bcn.cl/2015/index.php/Lonquimay\#N. C3.BAmero_de_cabezas_de_ganado_por_ especie_2007

33. Biblioteca del Congreso Nacional de Chile/ BCN. Reportes Estadísticos Comunales 2015 Melipeuco [Internet]. Valparaíso: BCN; 2015 [citad 3 Dic 2018]. [aprox. 36 pantallas]. Disponible en: https://reportescomunales.bcn. cl/2015/index.php/Melipeuco\#N.C3.BAmero_de_cabezas_de_ganado_por_especie_2007

34. Biblioteca del Congreso Nacional de Chile/ BCN. Reportes Estadísticos Comunales 2015
Curacautín [Internet]. Valparaíso: BCN; 2015 [citado 3 Dic 2018]. [apróx. 36 pantallas]. Disponible en: http://reportescomunales. bcn.cl/2015/index.php/Curacaut\%C3\%ADn\#N.C3.BAmero_de_cabezas_de_ganado_ por_especie_2007

35. Instituto Nacional de Estadísticas Chile. Censo de población y vivienda 2002 [Internet]. Santiago, Chile: INE; 2002 [citado 3 Dic 2018]. [aprox. 3 pantallas]. Disponible en: http://www.ine.cl/estadisticas/censos/censos-de-poblacion-y-vivienda

36. Departamento de Estadísticas e Información de Salud (DEIS). Enfermedades de Notificación Obligatoria (ENO). Santiago, Chile: Ministerio de Salud; 2014.

37. Heydari A, Khorashadizadeh F. Pender's health promotion model in medical research. JPMA The Journal of the Pakistan Medical Association. 2014; 64(9): 1067-74.

38. Ojodale PI, Umoh VJ, Abdullahi IO. Detection of Trichinella Antibodies in Slaughtered Pigs and Risk Factors Associated with Trichinellosis in Pig Farms in Kaduna Metropolis, Nigeria. Int J Life Sci Med Res [Internet]. 2015 Feb. [cited 2018 Oct 25]; 5(1): 1-6. Available from: http://www.academicpub.org/DownLoadPaper.aspx?paperid $=16574$

39. Singh BB, Kaur R, Gill G, Gill JPS, Soni RK, Aulakh RS. Knowledge, attitude and practices relating to zoonotic diseases among livestock farmers in Punjab, India: Acta Trop [Internet]. 2019 Jan [cited 2018 Dec 3]; 189: 15-21. Available from: doi: 10.1016/j.actatropica.2018.09.021. Epub 2018 Sep 27.

40. Franssen F, Swart A, van der Giessen J, Havelaar A, Takumi K. Parasite to patient: A quantitative risk model for Trichinella spp. in pork and wild boar meat. Int J Food Microbiol. 2017; 241: 262-75.

41. Ayral F, Artois J, Zilber AL, Widén F, Pounder KC, Aubert D et al. The relationship between socioeconomic indices and potentially zoonotic pathogens carried by wild Norway rats: a survey in Rhône, France (2010-2012). Epidemiol Infect [Internet]. 2015 Feb [cited 2018 Oct 26]; 143: 586-599. Available from: 
https://www.ncbi.nlm.nih.gov/pmc/articles/ PMC4411646/

42. Piarroux M, Piarroux R, Knapp J, Bardonnet K, Dumortier J, Watelet J et al. Populations at Risk for Alveolar Echinococcosis, France. Emerg Infect Dis [Internet]. 2013 May [cited 2018 Oct 25]; 19(5): 721-728. Available from: https://www.ncbi.nlm.nih.gov/pmc/articles/ PMC3647496/
43. Traub RJ, Pednekar RP, Cuttell L, Porter RB, Abd Megat Rani PA, Gatne ML. The prevalence and distribution of gastrointestinal parasites of stray and refuge dogs in four locations in India. Vet Parasitol [Internet]. 2014 Sept [cited 2018 Oct 25]; 205(1-2): 233 238. Available from: https://www.ncbi.nlm. nih.gov/pubmed/25139393 
Anexo. Encuesta Conocimiento y Práctica en Triquinosis, Hanta y Equinococosis Quística.

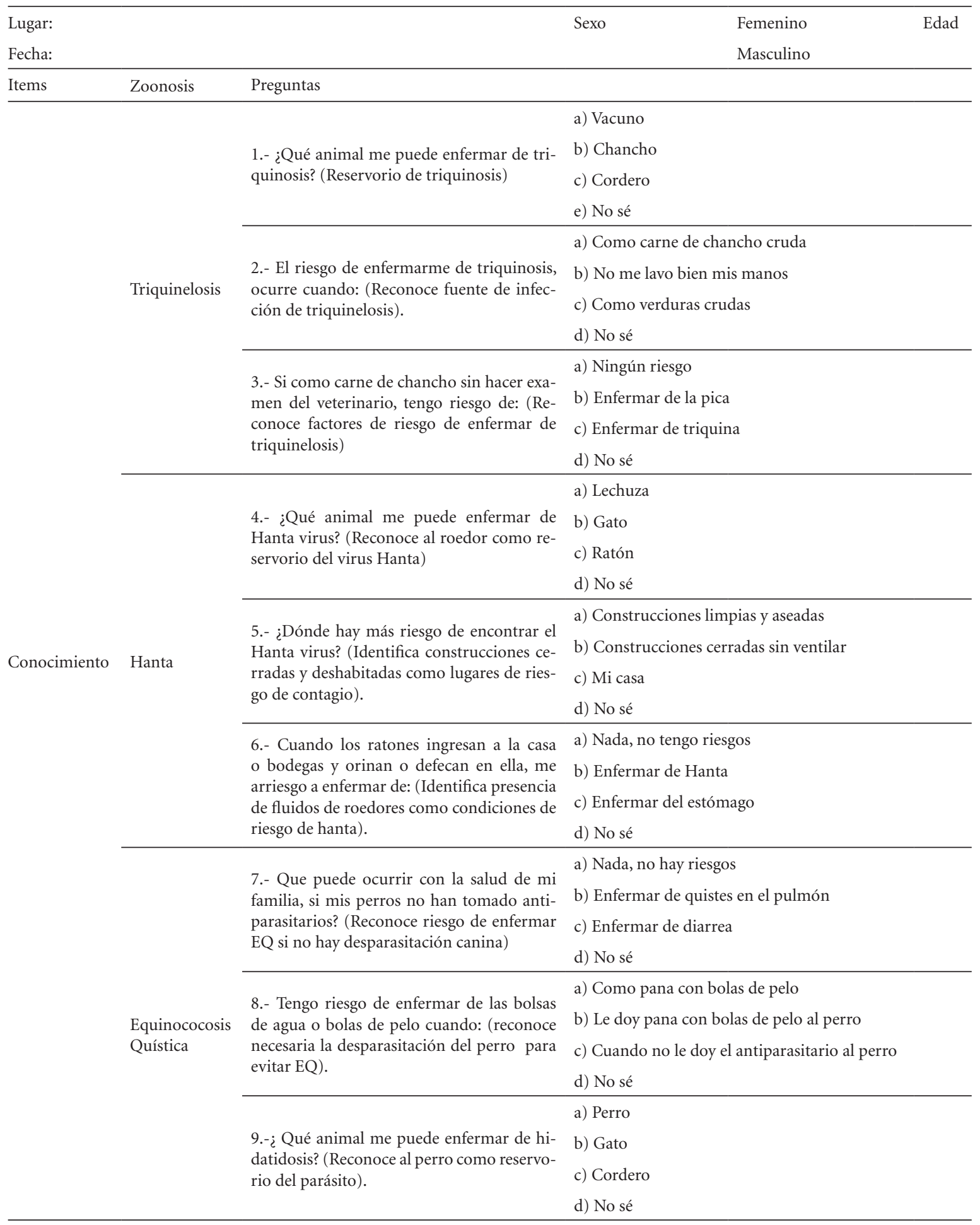


A continuación, se le solicita seleccionar la opción Muy de acuerdo, De acuerdo, No sé/ indiferente, En desacuerdo, según le parezcan las aseveraciones expuestas en relación a Triquinosis, Hanta e Hidatidosis.

\begin{tabular}{llll}
\hline Afirmación & Muy de acuerdo & De acuerdo & En desacuerdo \\
\hline
\end{tabular}

1a. La carne de cerdo la como siempre bien cocida (Come carne de cerdo bien cocida).

2a. El lugar donde comen los chanchos debe ser limpiado una vez a la semana (Mantiene los cerdos en lugares limpios).

3a. Siempre que faeno un chancho, le realizo el examen de la triquina con el veterinario (Realiza examen veterinario antes de consumir cerdo).

4a. Cuando consumo carne de lechones o chanchos nuevos no me enfermo de triquina (Reconoce al lechón como animal sin riesgo de contagiar triquinosis).

5a. El jugo de limón cuece la carne (Acepta que el jugo de limón cuece la carne)

6a. Con el jugo de limón puedo saber si la carne de chancho tiene triquina (Reconoce utilidad del uso de jugo de limón para identificar carne infectada con larvas de Trichinella spiralis).

7a. Cuando faeno animales, los restos no utilizados los boto en lugares fuera del alcance de los perros (Los restos orgánicos de animales faenados los dejan lejos del alcance del perro)

8a. Luego de acariciar a mi perro me lavo las manos (Se lava las manos después del contacto con perros).

Práctica

9a. A mi perro le doy la pastilla antiparasitaria una vez al año (La desparasitación del perro es necesaria para evitar esta enfermedad. Desparasita periódicamente al perro).

10a. Los quistes del pulmón, salen al respirar o inhalar pelos de perro (Conoce el mecanismo de transmisión de hidatidosis).

11a. Los gatos nos enferman de las bolas de pelo o bolsas de agua (Reconoce al gato como agente etiológico de hidatidosis).

12a. Cuando la pana de la oveja sale con bolsas de agua, se la doy al perro (Da vísceras contaminadas o crudas al perro).

13a. Cuando ingreso a bodegas deshabitadas, ventilo mínimo 30 minutos el lugar (Ventila construcciones antes de ingresar a ellas).

14a. Cuando ingreso a bodegas cerradas, me cubro la nariz con pañuelos o mascarilla (Se protege la vía aérea al ingresar a lugares cerrados).

15a. Desmalezo y limpio el entorno de mi casa para mantener el lugar libre de roedores (Controla malezas y entorno del hogar despejado para evitar roedores).

16a. Si no veo roedores o ratones alrededor de mi casa, no me contagio de Hanta virus. (Considera posibilidades de contraer Hanta aún si no observa roedores).

17a. Las culebras y lechuzas nos protegen del Hanta (Reconoce lechuzas y culebras como depredadores del reservorio).

18a. Quienes vivimos en el campo, no nos enfermamos del Hanta (Reconoce riesgo de enfermar por vivir en zonas rurales). 